Pacific Journal of Mathematics

BOUNDEDLY HOLOMORPHIC CONVEX DOMAINS 


\title{
BOUNDEDLY HOLOMORPHIC CONVEX DOMAINS
}

\author{
DoNG S. KIM
}

\begin{abstract}
A boundedly holomorphic convex domain is a holomorphically convex domain with respect to the algebra of bounded holomorphic functions in the domain. The followings are shown in this paper: In a Riemann domain, a boundedly holomorphic convex domain is a domain of bounded holomorphy. With some restrictions, the converse is true. The spectrum of the algebra $B$ of bounded holomorphic functions is an envelope of bounded holomorphy provided that the completion of $B$ with the topology of uniform convergence on compact subsets is stable under differentiation. Finally, Stein manifolds of bounded type are introduced.
\end{abstract}

Let $\left(X_{1}, A_{1}\right)$ and $\left(X_{2}, A_{2}\right)$ be complex (analytic) manifolds. A map $\alpha: X_{1} \rightarrow X_{2}$ said to be biholomorphic if $\alpha$ is a homeomorphism of $X_{1}$ onto $X_{2}$ and both $\alpha$ and $\alpha^{-1}$ are holomorphic. $\alpha$ is called a spread map if $\alpha$ is a locally biholomorphic. We denote a complex manifold $(X, A ; \alpha)$ with a spread map $\alpha$. A Riemann domain is a complex manifold which spreads in $\left(C^{n}, O\right)$. We denote $B(X)$ for the algebra of all bounded holomorphic functions on $X$.

Definition 1. Let $(X, A)$ be a complex manifold and $D$ be open in $X$. Let $B=B(D) . D$ is said to be boundedly holomorphic convex if $\operatorname{hull}_{B} K=\hat{K}_{B}=\left\{x \in D ;|f(x)| \leqq\|f\|_{K}\right.$ for all $\left.f \in B\right\}$ is compact provided $K$ is a compact subset of $D$.

An open set $D$ of $X$ is called a region of bounded holomorphy if there is an $f \in B(D)$ for which every boundary point of $D$ is a boundary singularity in the sense that $f$ has no bounded analytic continuation to any open neighborhood of any boundary point (see [5]).

The following natural questions arise; if boundedly holomorphic convex domains are domains of bounded holomorphy, and vice versa. The answer for the first is affirmative.

LEMMA 1. Let $\left\{\alpha_{n}\right\}$ be a sequence of complex numbers such that $\left|\alpha_{n}\right| \leqq 1$. And let $\left\{f_{n}\right\}$ be a sequence of bounded complex functions on a set $X$ such that $\sum\left|f_{n}(x)\right|$ converges uniformly on $x$. Then the infinite product

$$
f(x)=\prod_{n=1}^{\infty}\left(\alpha_{n}+f_{n}(x)\right)
$$

converges uniformly on $X$. 
THEOREM 1. Let $(X, A ; \alpha)$ be a Riemann domain. If it is boundedly holomorphic convex then it is a domain of bounded holomorphy.

Proof. Assume that $X$ is connected. Write $X=\mathrm{U}_{n=1}^{\infty} \hat{K}_{n}$, where $K_{n} \subset K_{n+1}$ and $\hat{K}_{n}=\left\{x \in X ;|f(x)| \leqq\|f\|_{K_{n}}\right.$ for all $\left.f \in B(X)\right\}$. Let $\left\{x_{k}\right\}$ be a countable dense set in $X$, and let $\Delta_{k}=\Delta\left(x_{k}, d\left(x_{k}\right)\right)$, where $d(x)=$ $\sup \{r ; \Delta(x, r)\} ; \Delta(x, r)$ is the neighborhood of $x$ which is homeomorphic by $\alpha$ onto a polydisc with center $\alpha(x)$ and radius $r$ in $C^{n}$. Let $\left\{S_{n}\right\}$ be a sequence

$$
\Delta_{1}, \Delta_{1}, \Delta_{2}, \Delta_{1}, \Delta_{2}, \Delta_{3}, \Delta_{1}, \Delta_{2}, \cdots
$$

such that each $\Delta_{k}$ occurs in $\left\{S_{n}\right\}$ infinitely many times. Take $y_{n} \in$ $S_{n}-\hat{K}_{n}$ and choose a function $g_{n} \in B(X)$ such that $\left\|g_{n}\right\|_{X}=1$ and $\left|g_{n}\left(y_{n}\right)\right|>\left\|g_{n}\right\|_{K_{n}}$. By taking sufficiently high power of $g_{n}$ we can find $f_{n}$ such that $\left\|f_{n}\right\|_{X}=1,\left|f_{n}\left(y_{n}\right)\right| \leqq 1 / 9$, and $\left\|f_{n}\right\|_{K_{n}}<1 /\left(n \cdot 2^{l_{n}}\right)$, where $l_{n} \geqq n$ and $l_{n} \in Z_{+}$. Also, by induction, we can arrange $l_{n+1}>l_{n}$. Put $f_{n}=(9 / 10) f_{n}$ (for simplicity use the same $f_{n}$ ), then $\left\|f_{n}\right\|_{2}=9 / 10$, $\left|f_{n}\left(y_{n}\right)\right| \leqq 1 / 10$, and $\left\|f_{n}\right\|_{K_{n}}<(9 / 10) \cdot\left(1 / n \cdot 2^{l_{n}}\right)$. Put $f_{n}\left(y_{n}\right)=\alpha_{n}$ and consider $\prod_{n=1}^{\infty}\left(\alpha_{n}-f_{n}\right)^{l_{n}}$. Since

$$
\sum_{n=1}^{\infty} n \cdot\left\|f_{n}\right\|_{K_{n}}<(9 / 10) \cdot 2^{-l_{n}}<\infty, \Pi_{n=1}^{\infty}\left(\alpha_{n}-f_{n}\right)^{l_{n}}
$$

converges uniformly on compact subsets of $X$ to a holomorphic function $f$. And since $\left\|\alpha_{n}-f_{n}\right\|_{x} \leqq 1, f \in B(X)$. Furthermore, $\left\|f_{n}\right\|_{K_{1}}<\alpha_{n}$ for all $n$ so that $\left(\alpha_{n}-f_{n}\right) \mid K_{1}$ is a unit of $C\left(K_{1}\right)$; the Banach algebra of continuous complex functions on $K_{1}$. Thus

$$
f\left|K_{1}=\lim _{\nu \rightarrow \infty} \prod^{\nu}\left(\alpha_{n}-f_{n}\right)^{l_{n}}\right| K_{1} ;
$$

the uniform limit in $C\left(K_{1}\right)$, is a unit of $C\left(K_{1}\right)$. Thus $f \neq 0$ in $X$.

Suppose for some $y \in X, \operatorname{rad}(f, y)>d(y)+\varepsilon$, where $\operatorname{rad}(f, y)$ is the radius of convergence of $f$ at $y$. Since $\left\{x_{k}\right\}$ is a dense subset of $X$ we can choose $x_{k}$ close enough to $y$ such that $\operatorname{rad}\left(f, x_{k}\right)>d\left(x_{k}\right)+$ $\varepsilon / 3$ and the power series $\hat{f}$ at $x_{k}$ converges in $\Delta\left(\alpha\left(x_{k}\right), d\left(x_{k}\right)+\varepsilon / 3\right)$. Since every $\Delta_{k}$ occurs in $\left\{S_{n}\right\}$ infinitely often $\Delta\left(x_{k}, d\left(x_{k}\right)\right)$ contains infinitely many points $y_{n}$, and $f$ has a zero of order $n$ at $y_{n}$. Choose $\left\{s_{n}\right\} \subset\left\{\alpha\left(y_{n}\right)\right\} \subset \Delta\left(\alpha\left(x_{k}\right), d\left(x_{k}\right)\right)$ such that $s_{n} \rightarrow s_{0} \in \bar{J}\left(\alpha\left(x_{k}\right), d\left(x_{k}\right)\right)$. Then, by continuity, for any $k_{1}, \cdots, k_{n}$

$$
\frac{\partial^{k_{1}+\cdots+k_{n}}}{\partial z_{1}^{k_{1}} \cdots \partial z_{n}^{k_{n}}} \hat{f}\left(s_{0}\right)=\lim _{n \rightarrow \infty} \frac{\partial^{k_{1}+\cdots+k_{n}}}{\partial z_{1}^{k_{1}} \cdots \partial z_{n}^{k_{n}}} \hat{f}\left(s_{n}\right)=0 .
$$

Hence the power series expansion $\hat{f}$ at $s_{0}$ is identically zero, so $\hat{f} \equiv 0$, which is a contradiction. Thus $\operatorname{rad}(f, y)=d(y)$ for all $y \in X$. Since $f \in B(X), X$ is a domain of bounded holomorphy. 
For the second question we raised; if a domain of bounded holomorphy is boundedly holomorphic convex, we first give a couple of examples of domains of bounded holomorphy which are not boundedly holomorphic convex.

ExAMPLE $10^{1}$ Let $D=\left\{(z, \omega) \in C^{2} ;|\omega|<e^{-|z|^{2}}\right\}$. If $f_{n} \in \mathcal{O}(C),\left|f_{n}\right| \leqq$ $k_{n} e^{n|z|^{2}}$ with $\Sigma k_{n}<\infty$, then $\Sigma f_{n}(z) \omega^{n}$ is bounded in $D$. It follows that $D$ is a domain of bounded holomorphy but it is not boundedly holomorphic convex.

ExAMPle 2. Let $D=\left\{(z, \omega) \in C^{2} ;|z|<|\omega|<1\right\}$. Then $D$ is a domain of bounded holomorphy but it is not boundedly holomorphic convex.

Thus we need some restrictions to claim an affirmative answer for the question.

Let $(X, A)$ be a complex manifold and $B=B(X)$. Denote $\widetilde{B}$ for the closure of $B$ in $C(X)$ with the topology of uniform convergence on compact subsets of $X$ (abbreviate as c.o. topology). If all partial derivatives of functions in $\widetilde{B}$ are in $\widetilde{B}, \widetilde{B}$ is said to be stable under differentiation.

Although the following propositions and their proofs are analogue to those for unbounded functions (see Katznelson [4] or Gunning and Rossi [3]), they have their own right for bounded functions.

Proposition 1. Let $(X, A ; \alpha)$ be a Riemann domain such that $d(K)<\infty$ for a compact subset $K$ of $X$, and let $B=B(X)$. Suppose $\widetilde{B}$ is stable under differentiation. Then (1) implies (2) and, in addition, if $X$ is finitely sheeted (2) implies (3).

(1) $(X, A ; \alpha)$ is a domain of bounded holomorphy,

(2) $d(K)=d\left(\hat{K}_{B}\right)$ for all compact subsets $K$ of $X$,

(3) $(X, A ; \alpha)$ is boundedly holomorphic convex.

NotE. If $D$ is merely a domain of holomorphy then (2) need not be true; let $D=\Delta(0,1)-\{0\}$, the punctured open unit disc in $C$, and $K$ be the circle with center 0 and radius $1 / 3$. Then $\hat{K}_{A}=K$ and $d(K)=d\left(\hat{K}_{A}\right)=1 / 3$. On the other hand $\hat{K}_{B}=\bar{A}(0,1 / 3)-\{0\}$ and $d\left(\widehat{K}_{B}\right)=0$.

Proposition 2. Let $(X, A ; \alpha)$ be a Riemann domain and $\alpha$ be a bounded spread map; i.e., $f_{1}, \cdots, f_{n} \in B(X)$ where $\alpha=\left(f_{1}, \cdots, f_{n}\right)$. Suppose that $B(X)$ separates the points of $X$. If $d(K)=d\left(\hat{K}_{B}\right)$ for all compact subsets $K$ of $X$ then $X$ is boundedly holomorphic convex.

\footnotetext{
${ }^{1}$ Example 1 is provided by the referee.
} 
Note. Let $D$ be a domain in Example 2 and $B=B(D)$. Then $\widetilde{B}$ is an algebra which is not stable under differentiation, for, if so $D$ is boundedly holomorphic convex by Propositions 1 and 2 .

Definition 2. Let $R\left(C^{n}, \mathscr{O}\right)$ be the category whose objects are complex manifolds $\left(X_{\nu}, A_{\nu} ; \alpha_{\nu}\right)$ spread in $\left(C^{n}, O\right)$ and morphisms are spread maps $\beta_{\mu}:\left(X_{\nu}, A_{\nu} ; \alpha_{\nu}\right) \rightarrow\left(X_{\mu}, A_{\mu} ; \alpha_{\mu}\right)$ such that $\alpha_{\nu}=\alpha_{\mu} \circ \beta_{\mu}$. Let $S(X, A ; \alpha, F)$ be the class of $\left(\beta_{\nu} ; X_{\nu}, A_{\nu} ; \alpha_{\nu}\right)$ such that $\beta_{\nu}:(X, A ; \alpha) \rightarrow$ $\left(X_{\nu}, A_{\nu} ; \alpha_{\nu}\right)$ is a morphism with $\alpha=\alpha_{\nu} \circ \beta_{\nu}$ and $\beta_{\nu}^{*} A_{\nu}\left(X_{\nu}\right) \supset F$, where $\beta_{\nu}^{*}$ is the adjoint of $\beta_{2}$. We define a quasi-order in $S(X, A ; \alpha, F)$ as follows: $\left(\beta_{\nu} ; X_{\nu}, A_{\nu} ; \alpha_{\nu}\right)<\left(\beta_{\mu} ; X_{\mu}, A_{\mu} ; \alpha_{\mu}\right)$ if and only if there exists a morphism $\gamma:\left(X_{\nu}, A_{\nu} ; \alpha_{\nu}\right) \rightarrow\left(X_{\mu}, A_{\mu} ; \alpha_{\mu}\right)$ such that $\beta_{\mu}=\gamma \circ \beta_{\nu}$ and $\alpha_{\nu}=$ $\alpha_{\mu} \circ \%$. With respect to this quasi-order there is a maximal object which is unique within biholomorphic morphism. We call this object the $F$-envelope of holomorphy of $(X, A ; \alpha)$. If $F=B(X)$ we call it the envelope of bounded holomorphy.

Note. The continuation of every function in $B(X)$ to the envelope is still bounded. To see this, apply the same argument on the envelope as in Lemma 15 of Kim [5].

A Bishop's theorem for an analytic structure on the spectrum of the algebra $A(X)$ carries in the following way over the bounded holomorphic functions. Although the proof is analogous to the case for unbounded functions we put it in detail. We are indebted for this proof to Quigley [7].

Let $(X, A)$ be a complex manifold and $B=B(X)$ with the c.o. topology. The spectrum $S_{B}$ of $B$ is the set of all nontrivial continuous complex homomorphisms of $B$ onto $C$.

Theorem 2. Let $(X, A ; \alpha)$ be a separable Riemann domain. Suppose $f_{i} \in B, i=1,2, \cdots, n$, where $\alpha=\left(f_{1}, \cdots, f_{n}\right)$, and $\widetilde{B}$ is stable under differentiation. Then $S_{B}$ is the envelope of bounded holomorphy.

Proof. We observe that $S_{B}=S_{\widetilde{B}}$ in set-wise and also topologically. Put $S=S_{B}$. Define $\hat{\alpha}: S \rightarrow C^{n}$ by $\hat{\alpha}(\varphi)=\left(\varphi\left(f_{1}\right), \cdots, \varphi\left(f_{n}\right)\right)$ for $\varphi \in S$, and $\rho: X \rightarrow S$ by $\rho(x)=\pi_{x}$, where $\pi_{x}(f)=f(x)$ for all $f \in B$. We will show that there is an analytic structure $\widetilde{A}$ on $S$ so that $(\rho ; S, \widetilde{A} ; \hat{\alpha}, \hat{B})$ is the envelope of bounded holomorphy for $(X, A: \alpha, B)$.

(i) We claim that for $\varphi \in S$ there is a compact set $K \subset X$ such that $|\varphi(g)| \leqq\|g\|_{K}$ for all $g \in B$. If it is not true, let $X=\bigcup_{n=1}^{\infty} K_{n}$, where $K_{n}$ are compact and $K_{n} \subset K_{n+1}$, then we have $g_{n} \in B$ such that $\left|\varphi\left(g_{n}\right)\right|=1$ and $\left\|g_{n}\right\|_{K_{n}}<2^{-n}$. Then $\Sigma g_{n}$ converges to an element of $\widetilde{B}$, but since $\varphi$ is continuous on $\widetilde{B}, \varphi\left(\Sigma g_{n}\right)=\Sigma \varphi\left(g_{n}\right)$. The latter series 
is not convergent, which is absurd. Thus there is a compact set $K$ such that $|\varphi(g)| \leqq\|g\|_{K}$. Denote this $K, K=K_{\varphi}$.

(ii) Let $\boldsymbol{P}_{\varphi}=\left\{s \in \boldsymbol{C}^{n}\right.$; $\left.\|s-\hat{\alpha}(\varphi)\|_{\infty}<d\left(K_{\varphi}\right)\right\}$ and with $\varepsilon, 0<\varepsilon<$ $d\left(K_{\varphi}\right)$ let $\boldsymbol{P}_{\varphi, \varepsilon}=\left\{s \in \boldsymbol{C}^{n}:\|s-\hat{\alpha}(\varphi)\|_{\infty}<\varepsilon\right\}$. For $f \in B, \varphi \in S$, and $s \in \boldsymbol{P}_{\varphi}$ we define the functional

$$
L(f, \varphi, s)=\Sigma j !^{-1} \varphi\left(\partial \frac{j}{\alpha} f\right)\left(s_{1}-\hat{\alpha}(\varphi)_{1}\right)^{j_{1}} \cdots\left(s_{n}-\hat{\alpha}(\varphi)_{n}\right)^{j_{n}},
$$

where

$$
\partial \frac{j}{\alpha} f=\frac{\partial_{\alpha}^{j_{1}+\cdots+j_{n}}}{\partial z_{1}^{j_{1}} \cdots \partial z_{n}^{j_{n}}}(f) \text { and } \quad j !=j_{1} \cdot j_{2} \cdots j_{n} .
$$

Then by a proposition in several complex variables and the continuity of $\varphi$,

$$
\left|\varphi\left(\partial \frac{j}{\alpha} f\right)\right| \leqq\left\|\partial \frac{j}{\alpha} f\right\|_{K_{\varphi}} \leqq \frac{j !}{\varepsilon^{j_{1}+\cdots+j_{n}}}\|f\|_{\left(K_{\varphi}\right) \varepsilon}
$$

for all $f \in B(X)$, where $\left(K_{\varphi}\right)_{\varepsilon}=\boldsymbol{U}\left\{\bar{P}(x, \varepsilon) ; x \in K_{\varphi}\right\}$. Hence $L(f, \varphi, z)$ converges uniformly in $z$ on $\overline{\boldsymbol{P}}_{\varphi, s}$ for all $f \in B$ so that $L(\cdot, \varphi, z)$ is analytic in $\boldsymbol{P}_{\varphi}$. In particular, $L(\cdot, \varphi, s)$ is continuous for all $\varphi \in S$ and $s \in \boldsymbol{P}_{\varphi} . \quad$ Furthermore, since $\varphi$ and $\partial(j / \alpha)$ are linear, $L(\cdot, \varphi, s): B \rightarrow \boldsymbol{C}$ is linear and by using Leibnitz' formula:

$$
k !^{-1} \partial \frac{k}{\alpha}(f \cdot g)=\sum_{i_{1}+j_{1}=K_{1}} \cdots \sum_{i_{n}+j_{n}=K_{n}} i !^{-1}\left(\partial \frac{i}{\alpha} f\right) j !\left(\partial \frac{j}{\alpha} g\right),
$$

we know that $L(\cdot, \varphi, s)$ is multiplicative. Therefore, $L(\cdot, \varphi, s) \in S$ for $\varphi \in S$ and $s \in \boldsymbol{P}_{\varphi}$. Let $\varphi_{s}=L(\cdot, \varphi, s)$, then $L(\cdot, \varphi, s)$ has the following properties;

(a) $\varphi_{\hat{\alpha}(\varphi)}=L(\cdot, \varphi, \hat{\alpha}(\varphi))=\varphi$

(b) $\varphi_{s}\left(f_{j}\right)=L\left(f_{j}, \varphi, s\right)=s_{j}, 1 \leqq j \leqq n$ for $\alpha=\left(f_{1}, \cdots, f_{n}\right)$

(c) $\hat{\alpha}\left(\varphi_{s}\right)=s$.

(iii) We topologize $S$ as follows: Let $Q_{\varphi}=\left\{\varphi_{s} ; s \in \boldsymbol{P}_{\varphi}\right\}$, and $Q_{\varphi, s}=$ $\left\{\varphi_{s}: s \in \boldsymbol{P}_{\varphi, s}\right\}$. Take the family $\left\{Q_{\varphi, \varepsilon} ; \varphi \in S, 0<\varepsilon d\left(K_{\varphi}\right)\right\}$ to be a subbase then this family gives a topology $\mathscr{T}$ on $S$. We show that $\mathscr{T}$ topology on $S$ is equivalent to the weak topology on $S$ induced by functions $\hat{f}$, where $\hat{f}$ is the Gelfand transform of $f$. First, $\mathscr{T}$-topology is finer than the weak topology: It suffices to show that all $\hat{f}$ in $\hat{B}$ are $\mathscr{T}$-continuous. For a given $\delta>0$ we must find $\varepsilon>0$ such that $\varphi_{s} \in Q_{\varphi, \varepsilon} \Rightarrow\left|\hat{f}\left(\varphi_{s}\right)-\hat{f}(\varphi)\right|<\delta$, or equivalently such that

$$
s \in \boldsymbol{P}_{\varphi, \varepsilon} \Rightarrow\left|\varphi_{s}(f)-\varphi(f)\right|<\delta,
$$

or equivalently such that

$$
s \in \boldsymbol{P}_{\varphi, \varepsilon} \Rightarrow \mid L(f, \varphi, s)-L(f, \varphi, \hat{\alpha}(\varphi) \mid<\delta .
$$


Let $h(z)=L(f, \varphi, z)-L(f, \varphi, \hat{\alpha}(\varphi))=L(f, \varphi, z)-\varphi(f)$; then $h$ is analytic in $\boldsymbol{P}_{\varphi}$; in particular, $h$ is continuous on $\boldsymbol{P}_{\varphi}$. Hence there exists $\varepsilon>0$ such that $s \in \boldsymbol{P}_{\varphi, \varepsilon} \Rightarrow|h(s)|<\delta$ which shows that $\hat{f} \in \hat{B}$ are $\mathscr{T}$-continuous. Secondly, the weak topology is finer than $\mathscr{T}$-topology: It suffices to show that for $\varphi_{s} \in Q_{\varphi, \varepsilon}$ there is an open neighborhood of $\varphi_{s}$ (w.r.t. the weak topology) which is contained in $Q_{\varphi, \varepsilon}$. We first observe that $\left\|\hat{\alpha}(h)-\hat{\alpha}\left(\varphi_{s}\right)\right\|_{\infty}=\left\|\left(h\left(f_{1}\right)-\varphi_{s}\left(f_{1}\right), \cdots, h\left(f_{n}\right)-\varphi_{s}\left(f_{n}\right)\right)\right\|_{\infty}$. Let $\varepsilon_{1}$ be the radius of maximal polydisc with center $\hat{\alpha}\left(\varphi_{s}\right)=s$ which is contained in $\boldsymbol{P}_{\varphi, \varepsilon}$. If $\left\|\hat{\alpha}(h)-\hat{\alpha}\left(\varphi_{s}\right)\right\|_{\infty}<\varepsilon_{1}$ then $\hat{\alpha}(h) \in \boldsymbol{P}_{\varphi, \varepsilon}$ and so $h=h_{\hat{\alpha}(h)} \in Q_{\varphi, \varepsilon}$. Now $\left\|\hat{\alpha}(h)-\hat{\alpha}\left(\varphi_{s}\right)\right\|_{\infty}<\varepsilon_{1}$ iff $\left|h\left(f_{i}\right)-\varphi_{s}\left(f_{i}\right)\right|<\varepsilon_{1}$ for $1 \leqq i \leqq n$. Hence the open neighborhood $U_{\varphi_{s}}=\left\{h \in S: \mid \hat{f}_{i}(h)-\hat{f}_{i}\left(\varphi_{s}\right)\right) \mid<$ $\left.\varepsilon_{1}, 1 \leqq i \leqq n\right\}$ is contained in $Q_{\varphi, \varepsilon}$, which shows $Q_{\varphi, \varepsilon}$ is open w.r.t. the weak topology.

(iv) Since $\hat{\alpha}\left(\varphi_{s}\right)=s, \hat{\alpha} \mid \varphi_{\varphi, \varepsilon}$ is one-to-one and $\hat{\alpha} \mid \varphi_{\varphi, \varepsilon}=\boldsymbol{P}_{\varphi, \varepsilon}$. And $\phi=\phi_{\hat{\alpha}(\varphi)} \in Q_{\varphi, \varepsilon}$. It follows that $\hat{\alpha}: S \rightarrow C^{n}$ is a local homeomorphism. Let $\widetilde{A}=\hat{\alpha}^{*} \mathcal{O}$ (the analytic structure on $S$ induced by $\hat{\alpha}$ ), then $(S, \tilde{A} ; \hat{\alpha})$ is a complex analytic manifold. Note that $\widehat{B}=\{\widehat{f} ; f \in B\} \subset \widetilde{A}$. For $\varphi \in S, s \in \boldsymbol{P}_{\varphi}$, we have $\left(\hat{\alpha} \mid Q_{\varphi}\right)^{-1}(s)=\varphi_{s}$ so that $\hat{f} \circ(\hat{\alpha} \mid Q)^{-1}(s)=$ $\hat{f}\left(\varphi_{s}\right)=\varphi_{s}(f)=L(f, \varphi, s)$. Thus $\hat{f} \circ\left(\hat{\alpha} \mid Q_{\varphi}\right)^{-1}(z)=L(f, \varphi, z)$ which is convergent in $\boldsymbol{P}_{\varphi}$.

(v) $(S, \widetilde{A} ; \hat{\alpha}, \hat{B})$ is a $B$-continuation of $(X, A ; \alpha, B)$. Let $\pi_{x}$ be defined by $\hat{f}\left(\pi_{x}\right)=f(x)$ for $x \in X, f \in B$. Then $\pi_{x} \in S$ and $K_{\pi_{x}}=\{x\}$. Define $\rho: X \rightarrow S$ by $\rho(x)=\pi_{x}$. If $T_{x}$ is the $\alpha$-polydisc of center $x$ and radius $d\left(K_{\pi_{x}}\right)=d(x)$ then $\alpha \mid T_{x}$ is one-to-one and $\alpha T_{x}=\boldsymbol{P}_{\pi_{x}}$. In fact $\hat{\alpha} \circ \rho(x)=\widehat{\alpha}\left(\pi_{x}\right)=\left(\pi_{x}\left(f_{1}\right), \cdots, \pi_{x}\left(f_{n}\right)\right)=\left(f_{1}(x), \cdots, f_{n}(x)=\alpha(x)\right.$ so that $\hat{\alpha} \circ \rho=\alpha$. We have shown that the following diagram commutes:

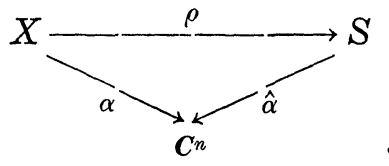

Now we have to show $\hat{f} \circ \rho=f$ for every $f \in B$. We first observe that for each $q \in T_{x}, L\left(\cdot, \pi_{x}, \alpha(q)\right)=\pi_{q}$. For $\hat{\alpha}\left(\pi_{x}\right)=\alpha(x)$ and so

$$
\begin{aligned}
L\left(f, \pi_{x}, \alpha(q)\right) & =\Sigma j !^{-1} \pi_{x}\left(\partial \frac{j}{\alpha} f\right)\left(\alpha(q)_{1}-\alpha(x)_{1}\right)^{j_{1}} \cdots\left(\alpha(q)_{n}-\alpha(x)_{n}\right)^{j_{n}} \\
& =\Sigma j !^{-1}\left(\partial \frac{j}{\alpha} f\right)(x)\left(\alpha(q)_{1}-\alpha(x)_{1}\right)^{j_{1}} \cdots\left(\alpha(q)_{n}-\alpha(x)_{n}\right)^{j_{n}} \\
& =f(q) \\
& =\pi_{q}(f) .
\end{aligned}
$$

$Q_{\pi_{x}}=\left\{\left(\pi_{x}\right)_{s} ; s \in \boldsymbol{P}_{\pi_{x}}\right\}=\left\{\pi_{q} ; q \in T_{x}\right\}$, so that $\rho$ maps $T_{x}$ one-to-one and onto $Q_{\pi_{x}}$. Thus $\rho: X \rightarrow S$ is a local homeomorphism, and for $f \in B$, $\hat{f} \circ \rho(x)=\hat{f}\left(\pi_{x}\right)=\pi_{x}(f)=f(x)$ for all $x \in X$. Hence $\hat{f} \circ \rho=f$ for all 


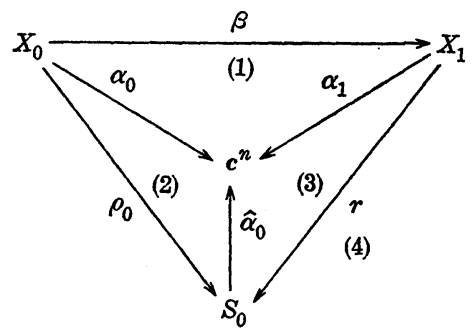

Figure 1

$f \in B$. Note that $\hat{f}$ is bounded on $S$ for all $f \in B$.

(vi) Let $X_{0}$ be a connected component of $X$ and $S_{0}$ be the connected component of $S$ containing $\rho X_{0}$. Let $f_{0}, \alpha_{0}, \rho_{0}, A_{0}, B_{0}$ be the restrictions of $f, \alpha, \rho, A, B$ to $X_{0}$ and $\hat{f}_{0}, \widehat{\alpha}_{0}, \widetilde{A}_{0}, \widehat{B}_{0}$ be the restrictions of $\hat{f}, \hat{\alpha}, \widetilde{A}, \hat{B}$ to $S_{0}$. Then $\left(S_{0}, \widetilde{A}_{0} ; \widehat{\alpha}_{0}, \hat{B}_{0}\right)$ is a $B_{0}$-continuation of $\left(X_{0}, A_{0}\right.$; $\left.\alpha_{0}, B_{0}\right)$. Moreover, we claim that $\left(S_{0}, \widetilde{A}_{0} ; \widehat{\alpha}_{0}, \widehat{B}_{0}\right)$ is a maximal $B$-continuation. Take $\left(\beta, X_{1}, A_{1} ; \alpha_{1}, B_{1}\right) \in S\left(X_{0}, A_{0} ; \alpha_{0}, B_{0}\right)$ such that image $\beta^{*} \supset B_{0}$, i.e., for $f_{0} \in B_{0}$ there is a unique $f_{1} \in B_{1}$ such that $f_{1} \circ \beta=f_{0}$ 。 To show the above diagrams commute:

Define $r: X_{1} \rightarrow S_{0}$ by $r\left(x_{1}\right)\left(f_{0}\right)=f_{1}\left(x_{1}\right), x_{1} \in X_{1}$. The commutativity of (1) is given and we have shown the commutativity of (2). To show (4), $r \circ \beta=\rho_{0}$; for $x_{0} \in X_{0}$ and $f_{0} \in B_{0}, r \circ \beta\left(x_{0}\right)\left(f_{0}\right)=f_{1}\left(\beta\left(x_{0}\right)\right)=f_{0}\left(x_{0}\right)=$ $\pi_{x_{0}}\left(f_{0}\right)=\rho_{0}\left(x_{0}\right)\left(f_{0}\right)$. Hence $r \circ \beta=\rho_{0}$. To show (3), $\widehat{\alpha}_{0} \circ r=\alpha_{1}$. Let $\alpha_{0}=\left(f_{10}, \cdots, f_{n 0}\right)$ and $\alpha_{1}=\left(f_{11}, \cdots, f_{n 1}\right)$ then $\hat{a}_{0} \circ r\left(x_{1}\right)=\left(r\left(x_{1}\right)\left(f_{10}\right), \cdots\right.$, $\left.r\left(x_{1}\right)\left(f_{n 0}\right)\right)=\left(f_{11}\left(x_{1}\right), \cdots, f_{n 1}\left(x_{n}\right)\right)$ for $x_{1} \in X_{1}$. It $x_{1} \in \beta\left(X_{0}\right)$, i.e., $x_{1}=\beta x_{0}$ for some $x_{0} \in X_{0}$ then $\hat{\alpha}_{0} \circ r\left(x_{1}\right)=\widehat{\alpha}_{0} r \beta\left(x_{0}\right)=\left(f_{11} \beta\left(x_{0}\right), \cdots, f_{n 1} \beta\left(x_{0}\right)\right)=$ $\left(f_{10}\left(x_{0}\right), \cdots, f_{n 0}\left(x_{0}\right)\right)=\alpha_{0}(x)=\alpha_{1} \beta\left(x_{0}\right)=\alpha_{1}\left(x_{1}\right)$. Thus $\hat{\alpha}_{0} \circ r=\alpha_{1}$ on $\beta X_{0} \subset$ $X_{1}$, and since $X_{1}$ is connected, $\hat{\alpha}_{0} \circ r=\alpha_{1}$ on $X_{1}$.

To show that $\left(S_{0}, \widetilde{A}_{0} ; \widehat{\alpha}_{0}, \widehat{B}_{0}\right)$ is $B$-continuation of $\left(X_{1}, A_{1} ; \alpha_{1}, \beta_{1}\right)$. For $x_{1} \in X_{1}$, let $r\left(x_{1}\right)=\varphi \in S_{0}$. Let $Q_{\varphi} \subset S_{0}$. Take an $\alpha_{1}$-polydisc $T_{1}$ of center $x_{1}$ such that $\alpha_{1} T_{1}=\boldsymbol{P}_{\varphi, \varepsilon}\left(\alpha_{1}\left(x_{1}\right)=\widehat{\alpha}_{0} \circ r\left(x_{1}\right)=\widehat{\alpha}_{0}(\varphi)\right.$ is the center). Then by (3), $r T_{1}=Q_{\varphi, \varepsilon}$ and $r \mid T_{1}$ is one-to-one since $\alpha_{1}\left|T_{1}, \hat{\alpha}\right| Q_{\varphi, \varepsilon}$ are one-to-one. Thus $r$ is a local homeomorphism. Now we know that $f_{1} \circ \beta=f_{0}=\hat{f}_{0} \circ \rho_{0}$, thus $\hat{f}_{0} \circ r \circ \beta=\hat{f}_{0} \circ \rho_{0}=f_{0}=f_{1} \circ \beta$. Hence $\hat{f}_{0} \circ r=f_{1}$ on $\beta X_{0} \subset X_{1}$. Since $X_{1}$ is connected $\hat{f}_{0} \circ r=f_{1}$ on $X_{1}$. We have shown that the following diagrams are commutative:

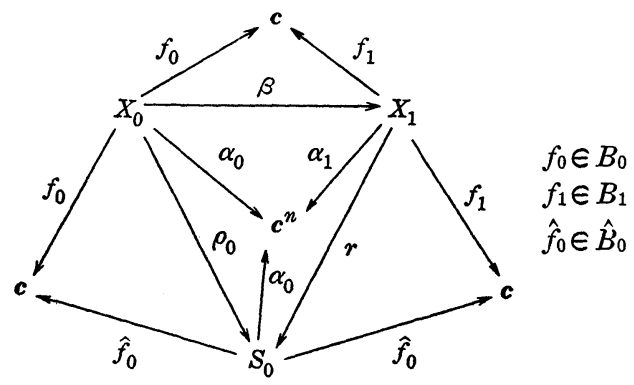

FIGURE 2 
It follows that $\left(S_{0}, \widetilde{A}_{0} ; \hat{\alpha}_{0}, \widehat{B}_{0}\right)$ is the $B$-envelope of holomorphy of $\left(X_{0}, \widetilde{A}_{0} ; \hat{\alpha}_{0}, \hat{B}_{0}\right)$.

(vii) Let $E$ be the union of connected components $S_{0}$ of $S$ such that $\rho X \cap S_{0} \neq \varnothing$. Then $(\rho ; E, \widetilde{A}|E ; \hat{\alpha}| E, \hat{B} \mid E)$ is the $B$-envelope of holomorphy of $(X, A ; \alpha, B)$. Finally we show that $E=S$; assume $\varphi \in S-E$. Choose $K_{\varphi}, K_{\varphi}$ compact in $X$. Let $\hat{B}_{\rho(K \varphi)}$ be the uniform closure of $\hat{B} \mid \rho\left(K_{\varphi}\right)$ in $\rho\left(\rho\left(K_{\varphi}\right)\right)$. Then $S_{K}=\operatorname{hull}_{\hat{B}} \rho\left(K_{\varphi}\right)$ is compact in its induced weak topology from $S$ and is the maximal ideal space of $\hat{B}_{\rho(K \varphi)}$. Since $E$ is open and closed, $S_{K \varphi} \cap E$ is open and closed in $S_{K_{\varphi}}$ and is compact. Then, by the Silov idempotent theorem there exists $\hat{g} \in \hat{B}_{\rho(K \varphi)}$ such that

$$
\widehat{g}(h)=\left\{\begin{array}{l}
1, h \in S_{K \varphi}-\left(S_{K \varphi} \cap E\right) \\
0, h \in S_{K \varphi} \cap E
\end{array}\right.
$$

since $\rho\left(K_{\varphi}\right) \subset S_{K \varphi} \cap E,\|\hat{g}\|_{\rho(K \varphi)}=0$ while $\hat{g}(\varphi)=1$. This is absurd. Thus $E=S$. The proof is complete.

Definition 3. A complex manifold $(X, A)$ is a Stein manifold of bounded type if it satisfies the following conditions:

(a) For every compact subset $K$ of $X, \hat{K}_{B}$ is compact,

(b) $B(X)$ separates the points of $X$,

(c) Every point of $X$ has a local coordinate system consisting of functions in $B(X)$.

EXAMPLES. Any analytic polyhedron in $\boldsymbol{C}^{n}$, in particular, a polydisc, is a Stein manifold of bounded type, so is an annulus in $\boldsymbol{C}$. A relatively compact domain $D$ in $C$ such that $D=\operatorname{int} \bar{D}$ is also a Stein manifold of bounded type.

A punctured open disc in $C$ is not a Stein manifold of bounded type, though it is a Stein manifold. Nor the domains in Examples 1 and 2.

If $(X, A)$ is a Stein manifold of bounded type, it is a Stein manifold. Moreover, by Theorem 4 in Ch. 9 of Cartan [2], $A(X)=\widetilde{B}(X)$. Conversely, if $(X, A)$ is a Stein manifold with $A(X)=\widetilde{B}(X)$, then it is a Stein manifold of bounded type.

We gather the above discussions in the following theorem.

Theorem 3. Let $(X, A ; \alpha)$ be a separable Riemann domain with a bounded spread map. Then (1) implies (2) and (2) implies (3):

(1) $(X, A ; \alpha)$ is a Stein manifold of bounded type.

(2) Every nonzero continuous homomorphism of $B(X)$ is the point evaluation of $X$.

(3) $(X, A ; \alpha)$ is a domain of bounded holomorphy. 
Furthermore, if $B(X)$ separates the points of $X$ and $\widetilde{B}(X)$ is stable under differentiation then (3) implies (1).

REMARK. For a domain $D$ of bounded holomorphy, $\widetilde{B}(D)$ need not be stable under differentiation; for instance, the domains in Examples 1 and 2.

Example 2 shows that even if $B(D)$ separates the points of $D, \widetilde{B}(D)$ is not stable under differentiation, consequently $\widetilde{B}(D) \nsubseteq \mathcal{O}(D)$.

The author wishes to thank Professor Frank D. Quigley for his suggestions.

\section{REFERENCES}

1. E. Bishop, Holomorphic completion, analytic continuation, and the interpolation of semi-norms, Ann. Math., 87 (1963).

2. H. Cartan, Seminaire Henri Cartan, 1951-52, Fonctions analytigues de plusieurs variables complexes, W. A. Benjamin, Inc. (1967).

3. Gunning and Rossi, Analytic Functions of Several Complex Variables, Prentice-Hall, 1965.

4. Y. Katznelson, Lectures on Several Complex Variables, Yale Lecture Note, 1963-1964.

5. D. S. Kim, Bounded holomorphic functions of several complex variables, Trans. Amer. Math. Soc., 158 p. 437-443.

6. P. Malgrange, Lectures on the Theory of Functions of Several Complex Variables, Tata Institute, Bombay, 1960.

7. F. Quigley, Lectures on Several Complex Variables, Tulane University, 1963-1966.

Received December 14, 1971 and in revised form July 7, 1972.

UNIVERSITY OF FLORIDA 



\section{PACIFIC JOURNAL OF MATHEMATICS}

\section{EDITORS}

D. Gilbarg and J. Milgram

Stanford University

Stanford, California 94305

\author{
R. A. Beaumont \\ University of Washington \\ Seattle, Washington 98105
}

J. DuGUNDJI

Department of Mathematics

University of Southern California

Los Angeles, California 90007

Richard ARENS

University of California

Los Angeles, California 90024

\section{ASSOCIATE EDITORS}
E. F. BECKENBACH
B. H. NeumanN
F. WOLF
K. YosHIDA

\section{SUPPORTING INSTITUTIONS}

UNIVERSITY OF BRITISH COLUMBIA

CALIFORNIA INSTITUTE OF TECHNOLOGY

UNIVERSITY OF CALIFORNIA

MONTANA STATE UNIVERSITY

UNIVERSITY OF NEVADA

NEW MEXICO STATE UNIVERSITY

OREGON STATE UNIVERSITY

UNIVERSITY OF OREGON

OSAKA UNIVERSITY

\author{
UNIVERSITY OF SOUTHERN CALIFORNIA \\ STANFORD UNIVERSITY \\ UNIVERSITY OF TOKYO \\ UNIVERSITY OF UTAH \\ WASHINGTON STATE UNIVERSITY \\ UNIVERSITY OF WASHINGTON
* * * *
AMERICAN MATHEMATICAL SOCIETY \\ NAVAL WEAPONS CENTER
}

The Supporting Institutions listed above contribute to the cost of publication of this Journal, but they are not owners or publishers and have no responsibility for its content or policies.

Mathematical papers intended for publication in the Pacific Journal of Mathematics should be in typed form or offset-reproduced, (not dittoed), double spaced with large margins. Underline Greek letters in red, German in green, and script in blue. The first paragraph or two must be capable of being used separately as a synopsis of the entire paper. The editorial "we" must not be used in the synopsis, and items of the bibliography should not be cited there unless absolutely necessary, in which case they must be identified by author and Journal, rather than by item number. Manuscripts, in duplicate if possible, may be sent to any one of the four editors. Please classify according to the scheme of Math. Rev. Index to Vol. 39. All other communications to the editors should be addressed to the managing editor, Richard Arens, University of California, Los Angeles, California, 90024.

50 reprints are provided free for each article; additional copies may be obtained at cost in multiples of 50 .

The Pacific Journal of Mathematics is issued monthly as of January 1966. Regular subscription rate: $\$ 48.00$ a year (6 Vols., 12 issues). Special rate: $\$ 24.00$ a year to individual members of supporting institutions.

Subscriptions, orders for back numbers, and changes of address should be sent to Pacific Journal of Mathematics, 103 Highland Boulevard, Berkeley, California, 94708.

PUBLISHED BY PACIFIC JOURNAL OF MATHEMATICS, A NON-PROFIT CORPORATION

Printed at Kokusai Bunken Insatsusha (International Academic Printing Co., Ltd.), 270, 3-chome Totsuka-cho, Shinjuku-ku, Tokyo 160, Japan. 


\section{Pacific Journal of Mathematics}

\section{Vol. 46, No. 2 December, 1973}

Christopher Allday, Rational Whitehead products and a spectral sequence of

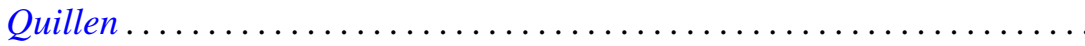

James Edward Arnold, Jr., Attaching Hurewicz fibrations with fiber

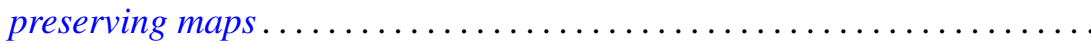

Catherine Bandle and Moshe Marcus, Radial averaging transformations with various metrics.................................

David Wilmot Barnette, A proof of the lower bound conjecture for convex

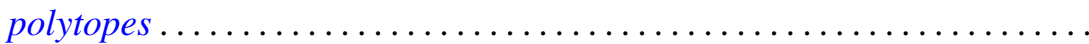

Louis Harvey Blake, Simple extensions of measures and the preservation of

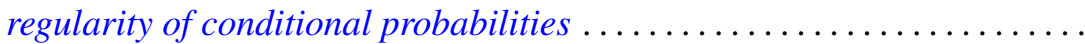

James W. Cannon, New proofs of Bing's approximation theorems for

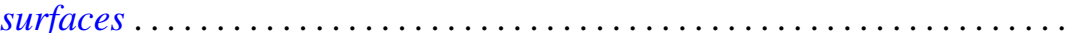

C. D. Feustel and Robert John Gregorac, On realizing HNN groups in

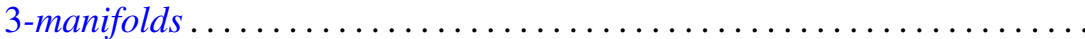

Theodore William Gamelin, Iversen's theorem and fiber algebras . . . . . . . . 389

Daniel H. Gottlieb, The total space of universal fibrations . . . . . . . . . . . .

Yoshimitsu Hasegawa, Integrability theorems for power series expansions of

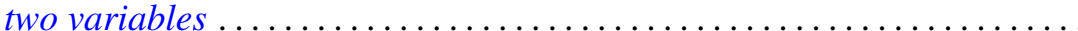

Dean Robert Hickerson, Length of period simple continued fraction expansion of $\sqrt{ } d$

Herbert Meyer Kamowitz, The spectra of endomorphisms of the disc algebra.

Dong S. Kim, Boundedly holomorphic convex domains

Daniel Ralph Lewis, Integral operators on $\mathscr{L}_{p}$-spaces ...

John Eldon Mack, Fields of topological spaces . . . . . . . . .

V. B. Moscatelli, On a problem of completion in bornology

Ellen Elizabeth Reed, Proximity convergence structures. .

Ronald C. Rosier, Dual spaces of certain vector sequence spaces .

Robert A. Rubin, Absolutely torsion-free rings

Leo Sario and Cecilia Wang, Radial quasiharmonic functions . .

James Henry Schmerl, Peano models with many generic classes .

H. J. Schmidt, The $\mathscr{F}$-depth of an $\mathscr{F}$-projector ............

Edward Silverman, Strong quasi-convexity. . . . . . . . . . . . . . . . . 549

Barry Simon, Uniform crossnorms ....................... 555

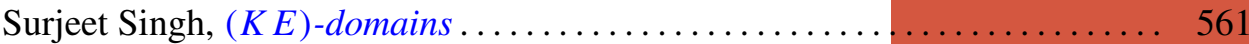

Ted Joe Suffridge, Starlike and convex maps in Banach spaces . . . . . . . . 575

Milton Don Ulmer, $C$-embedded $\Sigma$-spaces . . . . . . . . . . . . . . . . 591

Wolmer Vasconcelos, Conductor, projectivity and injectivity . . . . . . . . . 603 